\title{
Impact of Coronary Plaque Burden and Composition on Periprocedural Myocardial Infarction and Coronary Flow Reserve After Percutaneous Coronary Intervention
}

\author{
Yoshiharu Higuchi, ${ }^{1}$ MD, Takafumi Hiro, ${ }^{1}$ MD, Tadateru Takayama, ${ }^{1}$ MD, Takashi Kanai, ${ }^{1}$ MD, \\ Taro Kawano, ${ }^{1}$ MD, Daisuke Fukamachi, ${ }^{1}$ MD, Mitsumasa Sudo, ${ }^{1}$ MD, Toshihiko Nishida,,${ }^{1}$ MD, \\ Korehito Iida, ${ }^{1} \mathrm{MD}$, Satoshi Saito, ${ }^{1} \mathrm{MD}$, and Atsushi Hirayama, ${ }^{1} \mathrm{MD}$
}

\begin{abstract}
SUMMARY
Periprocedural myocardial infarction (PMI) is one of the major complications of percutaneous coronary intervention (PCI). We investigated the influence of coronary plaque burden and characteristics on PMI using intravascular ultrasound (IVUS) with radiofrequency-based tissue characterization technology (iMAP). The study population consisted of 33 consecutive patients with stable angina pectoris who underwent PCI. IVUS images were recorded before and after PCI for offline analysis, and coronary flow reserve (CFR) was measured after PCI. PMI was defined as a post-PCI cardiac troponin T elevation $>5 \times 99^{\text {th }}$ percentile of the upper reference limit $(0.014 \mathrm{ng} / \mathrm{mL})$. Plaque volume in patients with PMI $(n=12)$ was significantly greater than that in patients without PMI $(n=21)\left(240.4 \pm 106.0 \mathrm{~mm}^{3}\right.$ versus $152.1 \pm$ $\left.76.9 \mathrm{~mm}^{3}, P=0.0096\right)$. The iMAP-IVUS analysis demonstrated that the fibrotic, lipidic, and necrotic tissue volume within culprit lesions were also greater in patients with PMI than in patients without PMI $\left(129.4 \pm 52.2 \mathrm{~mm}^{3}\right.$ versus 94.6 $\pm 40.8 \mathrm{~mm}^{3}, P=0.041 ; 26.8 \pm 10.5 \mathrm{~mm}^{3}$ versus $15.8 \pm 11.5 \mathrm{~mm}^{3}, P=0.011$; and $81.3 \pm 48.4 \mathrm{~mm}^{3}$ versus $40.2 \pm 33.6$ $\mathrm{mm}^{3}, P=0.0071$, respectively). Multivariate logistic analysis demonstrated that necrotic tissue volume was the only independent predictor of PMI. Multiple regression analysis demonstrated that the post-PCI CFR values significantly correlated with percent plaque burden, and there were no correlations with the percent tissue burden of each plaque component. In conclusion, the iMAP-IVUS analyses demonstrate that necrotic tissue volume is a potent predictor of PMI. Microcirculatory disturbance after PCI is significantly influenced by percent plaque burden, regardless of plaque compositions. (Int Heart J 2014; 55: 391-396)
\end{abstract}

Key words: Intravascular ultrasound, Tissue characterization

$\mathrm{P}$ eriprocedural myocardial infarction (PMI) after percutaneous coronary intervention (PCI) is one of the major complications, despite progress in the development of techniques and device technologies. ${ }^{1,2)}$ A laboratory finding of PMI is defined as the release of cardiac troponin (cTn) $>5 \times$ $99^{\text {th }}$ percentile of the upper reference limit, ${ }^{3)}$ and cTn elevation after PCI is associated with increased adverse cardiac events. ${ }^{4-7)}$ Distal embolization is one of the strongest contributors to PMI, and embolic particles are frequently observed during PCI. ${ }^{1,8,9)}$ The amount of microembolization is strongly related to the magnitude of cTn elevation, microvascular dysfunction, and worsening of cardiac function. ${ }^{8-10}$

Myocardial microvascular function can be evaluated by quantifying the coronary flow reserve (CFR). ${ }^{11)} \mathrm{A}$ pressure/ temperature sensor-tipped guidewire can measure fractional flow reserve (FFR) and CFR by thermodilution. In the presence of normal FFR, a low CFR strongly suggests microvascular dysfunction. ${ }^{12)}$ Furthermore, coronary microembolization during PCI can cause a significant reduction in CFR, and the
CFR reduction can predict worse outcomes. ${ }^{11-14)}$

Because PMI occurs more frequently with PCI in patients with unstable angina pectoris, ${ }^{1)}$ plaque composition may contribute to the high probability of distal embolization. Radiofrequency-derived histopathological analysis by intravascular ultrasound (IVUS) has demonstrated that large necrotic cores are related to distal embolization and post-PCI cTn elevation. ${ }^{15,16)}$ On the other hand, some reports from IVUS studies have demonstrated that plaque burden is the strongest contributor to PMI. ${ }^{1,16,17)}$

Recently, new innovative radiofrequency-based analysis of tissue characterization by IVUS (iMAP-IVUS, Boston Scientific, Santa Clara, CA, USA) has become commercially available. The iMAP-IVUS can provide an accurate and reproducible method to characterize plaque tissue. ${ }^{18)}$

The purpose of the present study was to investigate the effect of plaque burden and tissue characteristics on post-PCI myocardial injury using iMAP-IVUS.

From the ${ }^{1}$ Division of Cardiology, Department of Medicine, Nihon University School of Medicine, Tokyo, Japan.

Address for correspondence: Yoshiharu Higuchi, MD, Division of Cardiology, Department of Medicine, Nihon University School of Medicine, 30-1 Ohyaguchi-kamicho, Itabashi-Ku, Tokyo 173-8610, Japan. E-mail: yhiguchi-ja@umin.net

Received for publication January 12, 2014. Revised and accepted February 7, 2014

Released in advance online on J-STAGE July 28, 2014.

All rights reserved by the International Heart Journal Association. 


\section{METHODS}

Study population: Thirty-three patients with stable angina pectoris who underwent PCI were prospectively enrolled in this study. The present study complied with the Declaration of Helsinki with regard to investigations of human subjects, and our hospital's ethical committee approved the study protocol. All patients provided written informed consent to participate in the study. The inclusion criteria were as follows: (1) no elevation of cardiac troponin $\mathrm{T}$ (cTnT) at admission, (2) a single de novo lesion, (3) a reference lumen diameter of $\geq 2.5 \mathrm{~mm}$, and (4) successful PCI with residual stenosis of $<25 \%$. The exclusion criteria were as follows: (1) a history of myocardial infarction or PCI, (2) side-branch occlusion by PCI, (3) use of a distal protection device, (4) chronic total occlusion, (5) dilated or hypertrophic cardiomyopathy, and (6) significant rhythm disturbance and valvular disease. Each patient was given 100 $\mathrm{mg}$ of aspirin and $75 \mathrm{mg}$ of clopidogrel $\geq 3$ days before PCI, and other cardiovascular medicines were continued in the present series of study procedures. The day after admission, coronary catheterization and PCI were performed using IVUS and a pressure/temperature sensor-tipped wire. The day after PCI, we tested blood samples to measure high-sensitivity cTnT to evaluate myocardial injury after PCI. PMI was arbitrarily defined as cTnT elevation $>5 \times 99^{\text {th }}$ percentile upper reference limit $(0.014 \mathrm{ng} / \mathrm{dL})$ in patients with normal baseline values. ${ }^{3)}$

PCI procedures and IVUS image acquisition: The PCI procedures were performed using coronary stents. Before the procedures, all patients were administered $100 \mathrm{IU} / \mathrm{kg}$ intravenous unfractionated heparin, and additional heparin boluses were administered to maintain an activated clotting time $>300 \mathrm{sec}-$ onds. Upon intracoronary administration of 100-300 $\mu \mathrm{g}$ of nitroglycerin, coronary angiography and subsequent IVUS observation were performed with a $2.6 \mathrm{Fr}$ 40-MHz Atlantis Pro IVUS catheter (Boston Scientific, Santa Clara, CA, USA) before balloon inflation. The IVUS transducer was positioned at least $20 \mathrm{~mm}$ distal to the target lesion, and was withdrawn by a motor at a speed of $0.5 \mathrm{~mm} / \mathrm{sec}$. Direct stenting was performed with a deployment pressure of 12 atmospheres, and subsequent post-balloon inflation was performed in cases with insufficient expansion or stent strut malapposition. IVUS observations were repeated after stent deployment, and the IVUS images were recorded for offline review. A quantitative measurement was performed according to the criteria of the American College of Cardiology Clinical Expert Consensus Document on Standards for Acquisition, Measurement and Reporting of Intravascular Ultrasound Studies. ${ }^{19)}$ Volumetric measurement and histopathological analyses of the culprit lesion at pre-PCI were performed using QIvus iMAP 2.0 software (Medis Medical Imaging System bv, Leiden, The Netherlands). A culprit lesion was defined as the segment that was treated with stents. PrePCI and post-stent IVUS images were reviewed side by side, and the segment that was treated with stents was identified on the pre-PCI IVUS imaging run. The stent-treated length was measured as the culprit lesion length, and the external elastic membrane (EEM) and lumen interface were traced semi-automatically on the pre-PCI IVUS images. The plaque volume was calculated as the EEM volume minus the lumen volume. The iMAP-IVUS analysis classified tissue segments within plaques into 4 major components according to the radiofrequency parameters: (1) light green was classified as fibrous tis- sue; (2) yellow was classified as lipidic tissue; (3) pink was classified as necrotic core; and (4) blue was classified as calcium. $^{20)}$

FFR and CFR analysis: Intracoronary FFR and CFR were measured with a 0.014-inch pressure/temperature sensortipped guidewire (PressureWire Certus, St. Jude Medical, Minneapolis, MN, USA). The PressureWire was advanced to position the pressure sensor at least $10 \mathrm{~mm}$ distal from the lesion. The central aortic pressure $(\mathrm{Pa})$ was measured through the guiding catheter tip, and the distal coronary pressure (Pd) was monitored. The FFR value was calculated as Pd divided by Pa. Next, a bolus of saline at room temperature (3 to $4 \mathrm{~mL}$ ) was briskly injected (less than 0.25 seconds) into the coronary artery. Three injections were administered, and the resting thermodilution curves were obtained to calculate the mean saline transit time at baseline (Tmn Bas). To induce maximal hyperemia, intravenous papaverine chloride $(8 \mathrm{mg}$ for the right coronary artery and $12 \mathrm{mg}$ for the left coronary artery) was administered. We then repeated the measurement of FFR and the mean saline transit time at hyperemia (Tmn Hyp). CFR was defined as the ratio of Tmn Hyp to Tmn Bas. ${ }^{21,22)}$

Statistical analyses: Continuous variables are presented as the mean \pm SD. Statistical analyses were performed using JMP 9 software (SAS Institute Inc., Cary, NC, USA). Student's $t$-test was used to compare continuous variables, and Pearson's chisquare analysis was used to compare categorical variables. A receiver-operator characteristic curve for the variable predicting PMI was generated from multiple sensitivity/specificity pairs. Multivariate logistic regression analysis was performed to identify which parameter obtained from iMAP-IVUS analysis was the independent predictor of PMI. Simple and multiple linear regression analyses were performed to assess the associations between iMAP-IVUS parameters and post-PCI CFR values. A value of $P<0.05$ was considered significant.

\section{RESUltS}

Patient characteristics and procedural results: The patients were divided into 2 groups according to the presence or absence of PMI: PMI $(+)$ group $(n=12)$ and PMI (-) group $(n=$ $21)$. There were no differences in baseline characteristics between the 2 groups (Table I). Lesion characteristics and procedural results are summarized in Table II. All patients in the PMI (-) group and 9 of 12 patients in the PMI (+) group were treated with one stent, and 3 of 12 patients among the PMI (+) patients were treated with two stents that overlapped one another with a small part of the stent edge. The length of the culprit lesion in the PMI (+) group was significantly greater than that in the PMI (-) group. Angiographic results of PCIs were successful in both groups, and the post-PCI FFR values in both groups were also successfully high. However, the post-PCI CFR values in the PMI (+) group were significantly lower than those in the PMI (-) group. These values indicated that PMI was accompanied by microcirculatory disturbance.

Gray-scale IVUS and iMAP-IVUS results: The conventional gray-scale IVUS findings are summarized in Table III. Volumetric analysis demonstrated that the plaque volume at prePCI in the PMI (+) group was significantly greater than that in the PMI (-) group. We then performed iMAP-IVUS analysis to identify tissue components of the plaque. Volumetric analyses 
Table I. Patient Characteristics at Baseline

\begin{tabular}{lccc}
\hline & PMI (+) group & PMI (-) group & $P$ \\
\hline Number & 12 & 21 & \\
Age (years) & $63 \pm 13$ & $69 \pm 10$ & 0.12 \\
Male gender, $n(\%)$ & $9(75)$ & $17(81)$ & 0.68 \\
Diabetes mellitus, $*$ (\%) & $9(75)$ & $12(57)$ & 0.31 \\
Hypertension, $n(\%)$ & $10(83)$ & $15(71)$ & 0.44 \\
Total cholesterol (mg/dL) & $180 \pm 42$ & $170 \pm 50$ & 0.57 \\
LDL cholesterol (mg/dL) & $102 \pm 19$ & $96 \pm 44$ & 0.68 \\
HDL cholesterol (mg/dL) & $46 \pm 11$ & $43 \pm 10$ & 0.51 \\
Triglycerides (mg/dL) & $176 \pm 114$ & $140 \pm 78$ & 0.50 \\
BUN (mg/dL) & $15.8 \pm 6.1$ & $13.8 \pm 3.6$ & 0.25 \\
Creatinine (mg/dL) & $0.95 \pm 0.46$ & $0.79 \pm 0.12$ & 0.16 \\
\hline
\end{tabular}

The data are presented as the mean \pm SD or number (\%) of subjects. LDL indicates low-density lipoprotein; HDL, high-density lipoprotein; and BUN, blood urea nitrogen. * Defined as hemoglobin Alc $\geq 6.2 \%$ and/or prescribed any diabetes therapy.

Table II. Lesion Characteristics and Procedural Results

\begin{tabular}{lccc}
\hline & PMI (+) group & PMI (-) group & $P$ \\
\hline Target Vessel, $n$ & & & \\
\multicolumn{1}{c}{ LAD / LCx / RCA } & $9 / 2 / 1$ & $8 / 5 / 8$ & 0.096 \\
Reference diameter (mm) & $2.8 \pm 0.5$ & $2.8 \pm 0.8$ & 0.67 \\
Minimal lumen diameter (mm) & $0.7 \pm 0.3$ & $0.6 \pm 0.3$ & 0.53 \\
Pre-PCI lesion stenosis (\%) & $79 \pm 9$ & $81 \pm 9$ & 0.62 \\
Post-PCI lesion stenosis (\%) & $3 \pm 5$ & $4 \pm 5$ & 0.69 \\
Stent diameter (mm) & $2.9 \pm 0.3$ & $3.1 \pm 0.3$ & 0.49 \\
Culprit lesion length (mm) & $30 \pm 9$ & $20 \pm 7$ & 0.0010 \\
Pre-PCI FFR values & $0.61 \pm 0.16$ & $0.64 \pm 0.20$ & 0.31 \\
Pre-PCI CFR values & $1.67 \pm 0.80$ & $1.79 \pm 0.73$ & 0.34 \\
Post-PCI FFR values & $0.92 \pm 0.06$ & $0.94 \pm 0.06$ & 0.36 \\
Post-PCI CFR values & $1.69 \pm 0.69$ & $2.92 \pm 1.29$ & 0.0087 \\
\hline
\end{tabular}

The data are presented as the mean \pm SD or number of subjects. LAD indicates left anterior descending coronary artery; LCx, left circumflex coronary artery; and RCA, right coronary artery.

Table III. Gray-scale IVUS Findings

\begin{tabular}{llll}
\hline & PMI (+) group & PMI (-) group & \multicolumn{1}{c}{$P$} \\
\hline EEM volume $\left(\mathrm{mm}^{3}\right)$ & $367.7 \pm 140.6$ & $259.4 \pm 114.6$ & 0.022 \\
Lumen volume $\left(\mathrm{mm}^{3}\right)$ & $127.3 \pm 47.5$ & $107.3 \pm 52.6$ & 0.29 \\
Plaque volume $\left(\mathrm{mm}^{3}\right)$ & $240.4 \pm 106.0$ & $152.1 \pm 76.9$ & 0.0096 \\
\hline
\end{tabular}

The data are presented as the mean \pm SD. EEM indicates external elastic membrane.

of absolute and relative plaque components are shown in Figure 1. Fibrotic, lipidic, and necrotic tissue volume were significantly greater in the PMI (+) group than in the PMI (-) group (Figure 1A). Although the percent necrotic tissue burden in the PMI (+) group was significantly greater than that in the PMI (-) group, the percent fibrotic tissue burden in the PMI (+) group was significantly smaller than that in the PMI (-) group. These findings suggested that the presence of PMI depended on the lesion vulnerability. Next, we sought to identify the independent predictors of PMI. Multivariate logistic regression analysis was performed to identify independent predictors of PMI. The multivariate model included the plaque volume, fibrotic tissue volume, lipidic tissue volume, necrotic tissue volume, percent fibrotic tissue burden, and percent necrotic tissue burden, in which significant differences were shown between 2 groups.
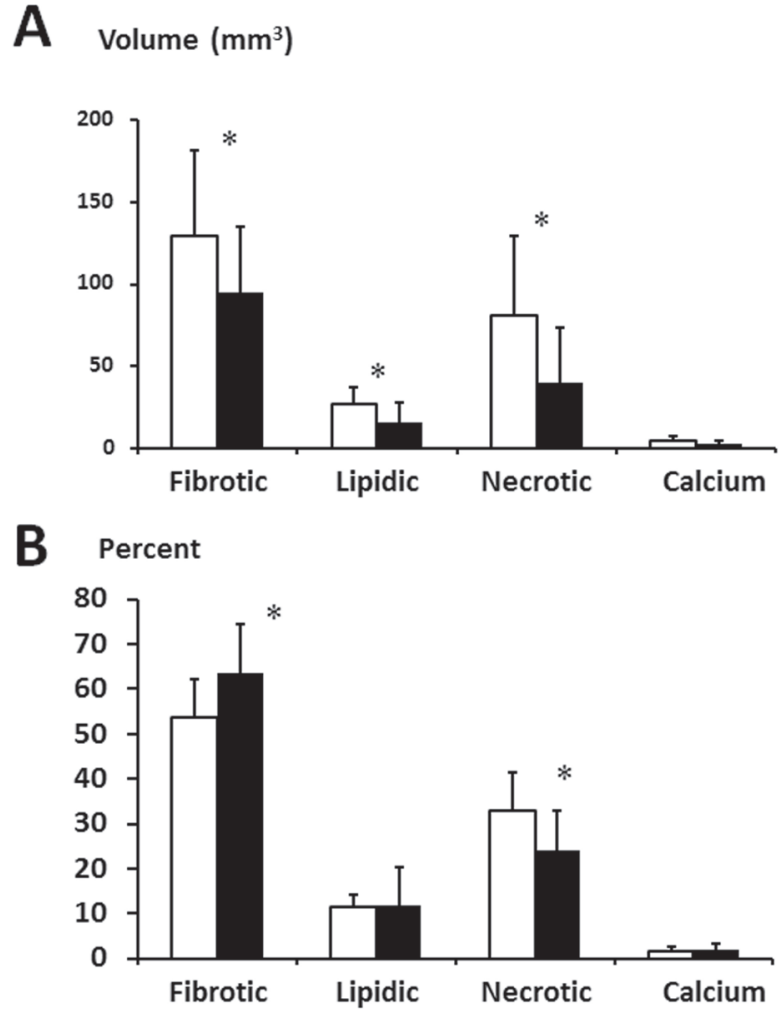

Figure 1. Volumetric analysis of absolute and relative plaque components. A: Comparison of absolute plaque volume between the 2 groups. The empty bars represent the PMI (+) group. The solid bars represent the PMI (-) group. The absolute fibrotic, lipidic, and necrotic tissue volumes were significantly greater in the PMI $(+)$ group than in the PMI (-) group $\left(129.4 \pm 52.2 \mathrm{~mm}^{3}\right.$ versus $94.6 \pm 40.8 \mathrm{~mm}^{3}, P=0.041 ; 26.8 \pm 10.5 \mathrm{~mm}^{3}$ versus $15.8 \pm 11.5 \mathrm{~mm}^{3}, P=0.011$; and $81.3 \pm 48.4 \mathrm{~mm}^{3}$ versus $40.2 \pm$ $33.6 \mathrm{~mm}^{3}, P=0.0071$, respectively). B: Comparison of relative tissue volumes between the 2 groups. The empty bars represent the PMI (+) group. The solid bars represent the PMI (-) group. The percent fibrotic tissue volume in the PMI (+) group was significantly smaller than that in the PMI (-) group $(54 \pm 9 \%$ versus $64 \pm 11 \%, P=0.017)$. The percent necrotic tissue volume in the PMI (+) group was significantly greater than that in the PMI (-) group $(33 \pm 9 \%$ versus $24 \pm 9 \%, P=0.014)$. There were no differences in percent lipidic tissue volume between the 2 groups (12 $\pm 3 \%$ versus 12 $\pm 8 \%, P=0.67)$. $* P<0.05$.

Table IV. Independent IVUS Predictor of PMI in Multivariate Analysis

\begin{tabular}{lccc}
\hline Variable & Odds ratio & $95 \% \mathrm{CI}$ & $P$ \\
\hline Necrotic tissue volume & 1.026 & $1.004-1.048$ & 0.020 \\
\hline
\end{tabular}

$\mathrm{CI}$ indicates confidence interval.

The necrotic tissue volume was the only independent predictor of PMI (Table IV). The receiver-operator characteristic curve for necrotic tissue volume predicting PMI is shown in Figure 2. A necrotic tissue volume of $50.93 \mathrm{~mm}^{3}$ was determined to be the optimal cut-off point to predict PMI with a sensitivity of $75 \%$ and a specificity of $71 \%$. The area under the curve was 0.779 .

Physiological measurements: Post-PCI CFR values in the PMI (+) group were significantly lower than those in the PMI (-) group despite the angiographic success and high post-PCI FFR 


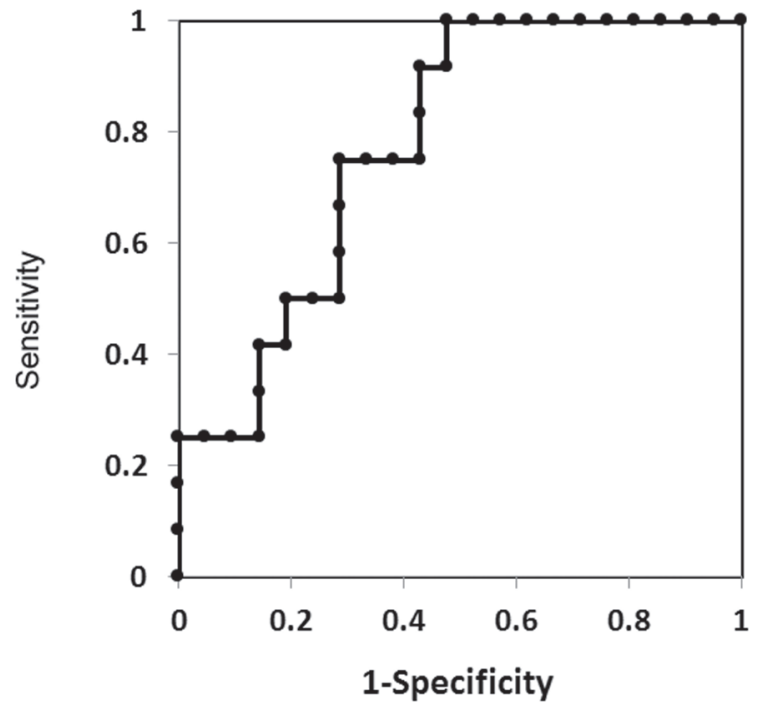

Figure 2. Receiver-operator characteristic curve for necrotic plaque volume predicting PMI. A necrotic tissue volume of $50.93 \mathrm{~mm}^{3}$ was determined to be the optimal cut-off point to predict PMI with a sensitivity of $75 \%$ and a specificity of $71 \%$. The area under the curve was 0.779 .

Table V. Correlations Between Post-PCI CFR Values and Absolute/Relative Plaque Volumes

\begin{tabular}{lll}
\hline Variable & $\mathrm{R}^{2}$ & $P$ \\
\hline Simple regression analysis & & \\
$\quad$ Plaque volume & 0.21 & 0.0099 \\
Fibrotic tissue volume & 0.19 & 0.017 \\
Lipidic tissue volume & 0.15 & 0.037 \\
Necrotic tissue volume & 0.19 & 0.015 \\
Calcified tissue volume & 0.063 & 0.18 \\
Percent plaque burden & 0.49 & 0.0006 \\
Percent fibrotic tissue burden & 0.049 & 0.24 \\
Percent lipidic tissue burden & 0.043 & 0.27 \\
Percent necrotic tissue burden & 0.076 & 0.14 \\
Percent calcified tissue burden & 0.079 & 0.13 \\
Multiple regression analysis & & \\
Percent plaque burden & 0.44 & 0.0061 \\
\hline
\end{tabular}

values in both groups. We then investigated the relationships between the post-PCI CFR values and plaque characteristics (Table V). Simple regression analyses demonstrated that the post-PCI CFR values had significant inverse correlations with the plaque volume, percent plaque burden, fibrotic tissue volume, lipidic tissue volume, and necrotic tissue volume. However, there were no correlations between the post-PCI CFR values and the percent tissue burden of each component. The percent plaque burden had a significant influence on the postPCI CFR value within the multiple regression analysis. The correlation between the post-PCI CFR values and the percent plaque burden is displayed in Figure 3. These findings suggested that microcirculatory disturbance depended on the percent plaque burden and was not associated with plaque composition.

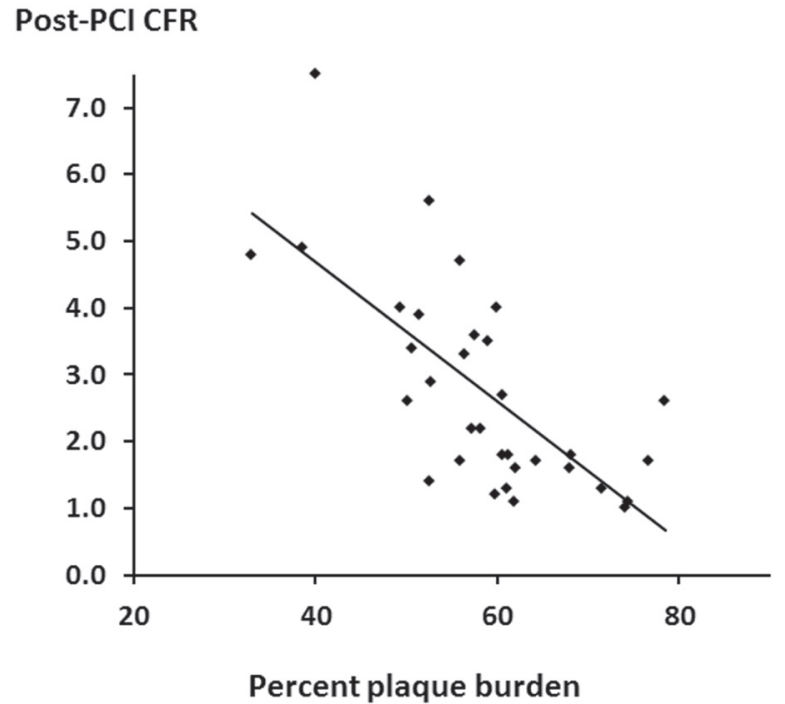

Figure 3. Correlation between post-PCI CFR and percent plaque burden. There was a significant inverse correlation between post-PCI CFR value and the percent plaque burden $\left(\mathrm{R}^{2}=0.49, P=0.0006\right)$.

\section{Discussion}

The present iMAP-IVUS study demonstrated that the necrotic tissue volume within culprit plaque was the only independent predictive factor of PMI. However, the microcirculatory disturbance after PCI depended on the percent plaque burden and did not correlate with plaque composition. These findings suggested that there were differences in the underlying mechanism between PMI and microcirculatory disturbance. In the context of no significant procedural complications, including coronary dissection or side-branch occlusion, PMI is considered to be caused by embolic particles of plaque debris and thrombus into the microcirculation during PCI. ${ }^{1)}$

In this study, we employed iMAP-IVUS to perform a plaque composition analysis. A recent iMAP-IVUS study revealed an inverse correlation between computed tomography (CT) intensity and the percentage of lipidic or necrotic tissue within plaque. ${ }^{23)} \mathrm{A}$ virtual histology (VH)-IVUS can also provide quantitative information on plaque composition using another algorithm of spectral analysis of IVUS radiofrequency data. ${ }^{20)}$ Hong, et al revealed that post-PCI cardiac troponin I elevation occurred in lesions with a large necrotic core area. ${ }^{16)}$ These studies support our findings that the necrotic tissue burden within plaque can predict PMI. Kawamoto, et al reported that the amount of necrotic core component identified with VH-IVUS was related to the amount of embolic particles during PCI. ${ }^{15)}$ From these findings, including our results, we suggest the possibility that atherosclerotic plaques containing a large necrotic core can easily liberate plaque debris or promote thrombus-formation during PCI.

The measurement of CFR is useful for investigating microcirculatory disturbance after PCI. In the present study, postPCI CFR values in the PMI (+) group were significantly lower than those in the PMI (-) group despite the angiographic success and high post-PCI FFR values in both groups. Previous studies have suggested mechanisms of a low CFR after PCI. First, the potential determinant of impaired post-PCI CFR was 
a low pre-PCI CFR, which was related to individual clinical conditions, including diabetes mellitus, hypertension, and myopathy. ${ }^{24)}$ In the present study, there was no difference in prePCI CFR values between the 2 groups and the percentages of patients with diabetes mellitus and hypertension were similar between the 2 groups. Second, other studies indicated there was a relationship between impaired CFR after PCI and microvascular obstruction. Herrmann, et al demonstrated that cTnT elevation was associated with a reduction of CFR, and the amount of plaque burden before PCI was related to the impairment of microcirculation. ${ }^{11)}$ Distal embolization of atherosclerotic debris is most likely the predominant pathophysiological mechanism leading to post-PCI microvascular obstruction. ${ }^{25)}$ Bahrmann, et al demonstrated that a large amount of embolic particles could reduce the post-PCI CFR. ${ }^{10)}$ In the present study, we excluded patients with side-branch occlusion after PCI, which could lead to low post-PCI CFR. We suggest that the procedural microembolization during PCI in the PMI (+) group reduced the post-PCI CFR, and therefore, the post-PCI CFR did not improve and remained as low as before PCI.

Our findings that microcirculatory disturbance after PCI is not correlated with plaque composition indicates that microcirculatory disturbance is more likely to occur after PCI compared with PMI. PMI is the result of irreversible necrotic change of myocytes, but microcirculatory disturbance is not necessarily accompanied by necrotic change of myocytes when the degree of disturbance is mild and transient. We hypothesize that the amount of embolic particles or debris burden liberated from necrotic cores is greater than that from other tissue components. The differences of the underlying causes between PMI and microcirculatory disturbance are likely to be dependent on the extent of necrotic tissue burden within the culprit lesion.

Study limitations: This study was a single-center study with a relatively small study population, which may raise the possibility of selection bias. Our study demonstrated that the plaque burden in the lesion has a potent influence on the probability of microcirculatory disturbance as well as PMI; however, there is no way to estimate the precise amount of liberated embolic debris burden during PCI. Because the fundamental mechanism of stenting procedures is to compress the plaque, the amount of liberated embolic debris burden is partly dependent on how easily the plaque is compressed, which may be related to plaque composition. Lastly, long-term follow-up prognostic implication data were not investigated in this study.

Clinical implications: To avoid PMI induced by downstream embolization, a number of mechanical and pharmacological approaches have already been suggested. ${ }^{1)}$ As for mechanical approach, distal protection devices may decrease myonecrosis after PCI. However, previous studies failed to demonstrate the benefit of a distal protection system during PCI for stable angina pectoris. ${ }^{1)}$ Disregarding an effect of lesion characteristics should be taken as 1 of the study limitations of those studies and the findings from the present iMAP-IVUS study might reconcile the controversy by considering the plaque characteristics during PCI. As for pharmacological approaches, several methods, including antiplatelet agents, anticoagulants, glycoprotein IIb/IIIIa inhibitors, statins, beta-blockers, and nicorandil, may decrease PMI. ${ }^{1)}$ Of those, statins and nicorandil can reduce the possibility of microvascular dysfunction after PCI. ${ }^{26,27)}$ If the lesion has the characteristics of large percent plaque burden and large necrotic tissue volume, the use of these drugs may potentially prevent PMI.

\section{REFERENCES}

1. Herrmann J. Peri-procedural myocardial injury: 2005 update. Eur Heart J 2005; 26: 2493-519. (Review)

2. Heusch G, Kleinbongard P, Böse D, et al. Coronary microembolization: from bedside to bench and back to bedside. Circulation 2009; 120: 1822-36. (Review)

3. Thygesen K, Alpert JS, Jaffe AS, Simoons ML, Chaitman BR, White HD. Third universal definition of myocardial infarction. J Am Coll Cardiol 2012; 60: 1581-98

4. Milani RV, Fitzgerald R, Milani JN, Lavie CJ. The impact of micro troponin leak on long-term outcomes following elective percutaneous coronary intervention. Catheter Cardiovasc Interv 2009; 74: 819-22.

5. Nienhuis MB, Ottervanger JP, Bilo HJ, Dikkeschei BD, Zijlstra F. Prognostic value of troponin after elective percutaneous coronary intervention: A meta-analysis. Catheter Cardiovasc Interv 2008; 71: 318-24. (Review)

6. Prasad A, Singh M, Lerman A, Lennon RJ, Holmes DR Jr, Rihal CS. Isolated elevation in troponin $\mathrm{T}$ after percutaneous coronary intervention is associated with higher long-term mortality. J Am Coll Cardiol 2006; 48: 1765-70.

7. Ricciardi MJ, Davidson CJ, Gubernikoff G, et al. Troponin I elevation and cardiac events after percutaneous coronary intervention. Am Heart J 2003; 145: 522-8.

8. Okamura A, Ito H, Iwakura K, et al. Detection and quantification of embolic particles during percutaneous coronary intervention to stable plaque: it correlates to coronary flow dynamics and myocardial damage. Catheter Cardiovasc Interv 2007; 69: 425-31.

9. Higuchi Y, Iwakura K, Okamura A, et al. Effect of embolic particles during coronary interventional procedures on regional wall motion in patients with stable angina pectoris. Am J Cardiol 2012; 109: 1142-7.

10. Bahrmann P, Werner GS, Heusch G, et al. Detection of coronary microembolization by Doppler ultrasound in patients with stable angina pectoris undergoing elective percutaneous coronary interventions. Circulation 2007; 115: 600-8.

11. Herrmann J, Haude M, Lerman A, et al. Abnormal coronary flow velocity reserve after coronary intervention is associated with cardiac marker elevation. Circulation 2001; 103: 2339-45.

12. Kini AS, Kim MC, Moreno PR, Krishnan P, Ivan OC, Sharma SK. Comparison of coronary flow reserve and fractional flow reserve in patients with versus without diabetes mellitus and having elective percutaneous coronary intervention and abciximab therapy (from the PREDICT Trial). Am J Cardiol 2008; 101: 796-800.

13. Ma J, Qian J, Ge J, et al. Changes in left ventricular ejection fraction and coronary flow reserve after coronary microembolization. Arch Med Sci 2012; 8: 63-9.

14. Albertal M, Voskuil M, Piek JJ, et al. Coronary flow velocity reserve after percutaneous interventions is predictive of periprocedural outcome. Circulation 2002; 105: 1573-8.

15. Kawamoto T, Okura H, Koyama Y, et al. The relationship between coronary plaque characteristics and small embolic particles during coronary stent implantation. J Am Coll Cardiol 2007; 50: 1635-40.

16. Hong YJ, Mintz GS, Kim SW, et al. Impact of plaque composition on cardiac troponin elevation after percutaneous coronary intervention: an ultrasound analysis. JACC Cardiovasc Imaging 2009; 2: 458-68.

17. Shibuya M, Okamura A, Hao H, et al. Prediction of distal embolization during percutaneous coronary intervention for unstable plaques with grayscale and integrated backscatter intravascular ultrasound. Catheter Cardiovasc Interv 2013; 81: E165-72.

18. Sathyanarayana S, Carlier S, Li W, Thomas L. Characterisation of atherosclerotic plaque by spectral similarity of radiofrequency intravascular ultrasound signals. EuroIntervention 2009; 5: 133-9. 
19. Mintz GS, Nissen SE, Anderson WD, et al. American College of Cardiology Clinical Expert Consensus Document on Standards for Acquisition, Measurement and Reporting of Intravascular Ultrasound Studies (IVUS). A report of the American College of Cardiology Task Force on Clinical Expert Consensus Documents. J Am Coll Cardiol 2001; 37: 1478-92. (Review)

20. Garcia-Garcia HM, Gogas BD, Serruys PW, Bruining N. IVUSbased imaging modalities for tissue characterization: similarities and differences. Int J Cardiovasc Imaging 2011; 27: 215-24. (Review)

21. Barbato E, Aarnoudse W, Aengevaeren WR, et al. Validation of coronary flow reserve measurements by thermodilution in clinical practice. Eur Heart J 2004; 25: 219-23.

22. Pijls NHJ, De Bruyne B, Smith L, et al. Coronary thermodilution to assess flow reserve: validation in humans. Circulation 2002; 105: 2482-6.

23. Utsunomiya M, Hara H, Moroi M, Sugi K, Nakamura M. Relationship between tissue characterization with $40 \mathrm{MHz}$ intravascu- lar ultrasound imaging and 64-slice computed tomography. J Cardiol 2011; 57: 297-302.

24. Kern MJ, Puri S, Bach RG, et al. Abnormal coronary flow velocity reserve after coronary artery stenting in patients: role of relative coronary reserve to assess potential mechanisms. Circulation 1999; 100: 2491-8

25. Jaffe R, Dick A, Strauss BH. Prevention and treatment of microvascular obstruction-related myocardial injury and coronary no-reflow following percutaneous coronary intervention: a systematic approach. JACC Cardiovasc Interv 2010; 3: 695-704. (Review)

26. Fujii K, Kawasaki D, Oka K, et al. The impact of pravastatin pretreatment on periprocedural microcirculatory damage in patients undergoing percutaneous coronary intervention. JACC Cardiovasc Interv 2011; 4: 513-20.

27. Hirohata A, Yamamoto K, Hirose E, et al. Nicorandil prevents microvascular dysfunction resulting from PCI in patients with stable angina pectoris: a randomised study. EuroIntervention 2014; 9: 1050-6. 